\title{
Clinical and economic outcomes associated with treatment sequences in patients with $B R A F$-mutant advanced melanoma
}

\author{
Ahmad Tarhini*,1, David McDermott ${ }^{2}$, Apoorva Ambavane ${ }^{3}$, Komal Gupte-Singh4, Valerie \\ Aponte-Ribero ${ }^{3}$, Corey Ritchings ${ }^{4}$, Agnes Benedict ${ }^{5}$, Sumati Rao ${ }^{4}$, Meredith M Regan ${ }^{6}$ \& \\ Michael Atkins ${ }^{7}$ \\ ${ }^{1}$ Department of Hematology \& Oncology, Cleveland Clinic, Taussig Cancer Institute, Cleveland, OH, 44106, USA \\ ${ }^{2}$ Beth Israel Deaconess Medical Center, Boston, MA, 02215, USA \\ ${ }^{3}$ Evidera, Inc., London, UK \\ ${ }^{4}$ Bristol-Myers Squibb, Princeton, NJ, 08648, USA \\ ${ }^{5}$ Evidera, Inc., Budapest, Hungary \\ ${ }^{6}$ Dana-Farber Cancer Institute, Harvard Medical School, Boston, MA, 02215, USA \\ ${ }^{7}$ Georgetown-Lombardi Comprehensive Cancer Center, Washington, DC, 20007, USA \\ *Author for correspondence: Tel.: +1 216636 0200; Fax: +1 216444 9464; tarhina1@ccf.org
}

\begin{abstract}
Aim: The cost-effectiveness of treatment sequences in BRAF-mutant advanced melanoma. Materials \& methods: A discrete event simulation model was developed to estimate total costs and health outcomes over a patient's lifetime (30 years). Efficacy was based on the CheckMate 067/069 trials and a matchingadjusted-indirect comparison between immuno-oncology and targeted therapies. Safety, cost (in US dollars; US third-party payer perspective) and health-related quality-of-life inputs were based on published literature. Results: Estimated survival gain was higher for sequences initiating with anti-PD-1 + anti-CTLA4 than for anti-PD-1 monotherapy or BRAF+MEK inhibitors. The incremental cost-effectiveness ratio per QALY gained for first-line anti-PD-1 + anti-CTLA-4 was US $\$ 54,273$ versus first-line anti-PD-1 and $\$ 79,124$ versus first-line BRAF+MEK inhibitors. Conclusion: Initiating treatment with anti-PD-1 + anti-CTLA-4 was more cost-effective than initiation with anti-PD-1 monotherapy or BRAF+MEK inhibitors.
\end{abstract}

First draft submitted: 19 October 2018; Accepted for publication: 3 December 2018; Published online: 19 December 2018

Keywords: anti-CTLA-4 + anti-PD-1 • checkpoint inhibition • treatment sequences

Approximately 1.2 million people in the USA were estimated to be living with a diagnosis of melanoma in 2014, with an estimated 91,270 new cases of invasive melanoma expected to be diagnosed in 2018. Further, an estimated 9320 will die from melanoma in 2018 [1]. Two main classes of drugs are approved for the treatment of patients with advanced melanoma: immuno-oncology therapies and targeted therapies [2]. Immuno-oncology therapies approved by the US FDA for the treatment of patients with advanced melanoma include ipilimumab (a cytotoxic T lymphocyte antigen-4 [CTLA-4] inhibitor) [3], pembrolizumab and nivolumab (programmed death 1 [PD-1] inhibitors) [4-7] and the combination of nivolumab plus ipilimumab [8,9]. Targeted therapies approved for the treatment of patients with advanced melanoma with a BRAF mutation ( $\sim 40-50 \%$ of all advanced melanoma patients) include vemurafenib [10] and dabrafenib [11] as monotherapy BRAF inhibitors and combination BRAF + MEK inhibitors (vemurafenib plus cobimetinib [12], dabrafenib plus trametinib [13-15] and encorafenib plus binimetinib [16]).

Long-lasting, durable antitumor immune responses are often observed in patients who respond to immunooncology agents [17]. In the CheckMate 067 trial, at 48-month follow up, the median duration of response was 50.1 months (95\% CI: 44.0 to not reached) for nivolumab + ipilimumab, was not reached (95\% CI: 45.7 to not reached) for nivolumab and was 14.4 months (95\% CI: 8.3 to not reached) with ipilimumab alone [18]. Durable responses were observed even among patients with early treatment discontinuation, leading to a potentially long treatment-free interval [19]. In a patient-level simulation study that included patients with advanced melanoma

Future Medicine 
from CheckMate 067 and 069 trials, the mean treatment-free interval was 1.9 years longer with nivolumab plus ipilimumab (5.3 years) than with nivolumab alone (3.4 years) and 3 years longer than with ipilimumab alone (2.3 years) [20]. The long-term benefits of immuno-oncology therapies are reflected in higher overall survival probabilities after 4 years of follow-up with plateauing (or 'tail') of the survival curves. In the CheckMate 067 trial, overall survival rates at 4 years were $53 \%$ with nivolumab plus ipilimumab, $46 \%$ with nivolumab alone and $30 \%$ with ipilimumab alone [18]. Notably, the overall survival rates were higher in patients with $B R A F$-mutant melanoma: $62 \%$ with nivolumab plus ipilimumab, 50\% with nivolumab and 33\% with ipilimumab, which could partly be explained by the salvage treatment impact of BRAF + MEK inhibitors available for these patients.

In clinical studies that used targeted therapies for patients with $B R A F$-mutant melanoma, the initial response rates with BRAF + MEK inhibitors were high $(50-70 \%)[13,14]$, but patients often acquired resistance, with the median response duration reported as 10-14 months [2,21]. The benefit of first-line targeted therapies is noted by early improvements in the progression-free survival and overall survival curves; however, a small subset of patients appear to experience long-term survival and experiencing a treatment-free period is uncommon. For combination therapy with dabrafenib plus trametinib, the 3-year overall survival rates in the COMBI-d [22,23] and COMBI-v [24] studies were approximately 44 and $45 \%$, respectively, and the progression-free survival rates were 22 and $25 \%$, respectively [23,24]. For those patients who stopped therapy while in response, the median time to progression was less than 6 months [23].

Given the lack of head-to-head clinical trial data, it is difficult to estimate the comparative effectiveness between treatment sequences initiated with targeted agents and those initiated with immuno-oncology agents for patients with $B R A F$-mutant advanced melanoma. In this regard, a clinical trial to assess the optimal treatment sequence of immuno-oncology and targeted agents in patients with BRAF-mutant melanoma is ongoing (NCT02224781), with an estimated completion date in 2022 [25].

Using published clinical trial results, analytical approaches can be used to extrapolate data over long-term periods to estimate health outcomes and costs associated with various treatment sequences. Such an approach was adopted in our recent study, in which individual patient simulation was used to evaluate the overall quality-adjusted life-years and total costs associated with relevant treatment sequences in patients with advanced melanoma and wild-type $B R A F$ [26]. In this current study, our objective was to assess the cost-effectiveness of select treatment sequences for patients with advanced melanoma and mutant $B R A F$. Specifically, we evaluated the optimal role of immunooncology agents and targeted therapies as first- or second-line treatment, based on their estimated health benefits and cost consequences.

\section{Materials \& methods}

\section{Model overview}

A patient-level simulation model was developed to estimate the incidence of various disease milestones and the associated total costs and health outcomes over the patient's lifetime (30 years) for treatment-naive BRAF-mutant advanced melanoma. Similar time horizons were used in other models for advanced melanoma studies [27-30]. Such a simulation model allowed for the incorporation of detailed clinical trial data to evaluate clinical outcomes, based on baseline patient characteristics and their changes over time. The model was based on pooled patient-level data from the Phase III CheckMate 067 [8,31] and Phase II CheckMate 069 [32,33] immuno-oncology clinical trials and published clinical trial data from COMBI-v $[13,24]$ and COMBI-d $[22,23]$ trials that evaluated BRAF + MEK targeted agents [34]. The treatment pathway was modeled in patients after diagnosis of advanced melanoma.

Three sequences for first- and second-line treatment of $B R A F$-mutant advanced melanoma were included in this study:

- First-line anti-PD-1 + anti-CTLA-4; second-line BRAF + MEK inhibitors

- First-line anti-PD-1; second-line BRAF + MEK inhibitors

- First-line BRAF + MEK inhibitors; second-line anti-PD-1

Treatment sequences that are representative of current practice were selected based on treatment guidelines, clinical relevance, market research and data availability [35,36]. Among the FDA-approved immuno-oncology therapies, nivolumab, pembrolizumab and nivolumab plus ipilimumab are widely used in the treatment of $B R A F$-mutant melanoma. Of the FDA-approved targeted agents for melanoma, the dabrafenib plus trametinib combination is more commonly used than the other targeted agents [37]. Sequences were modeled using data from nivolumab plus 


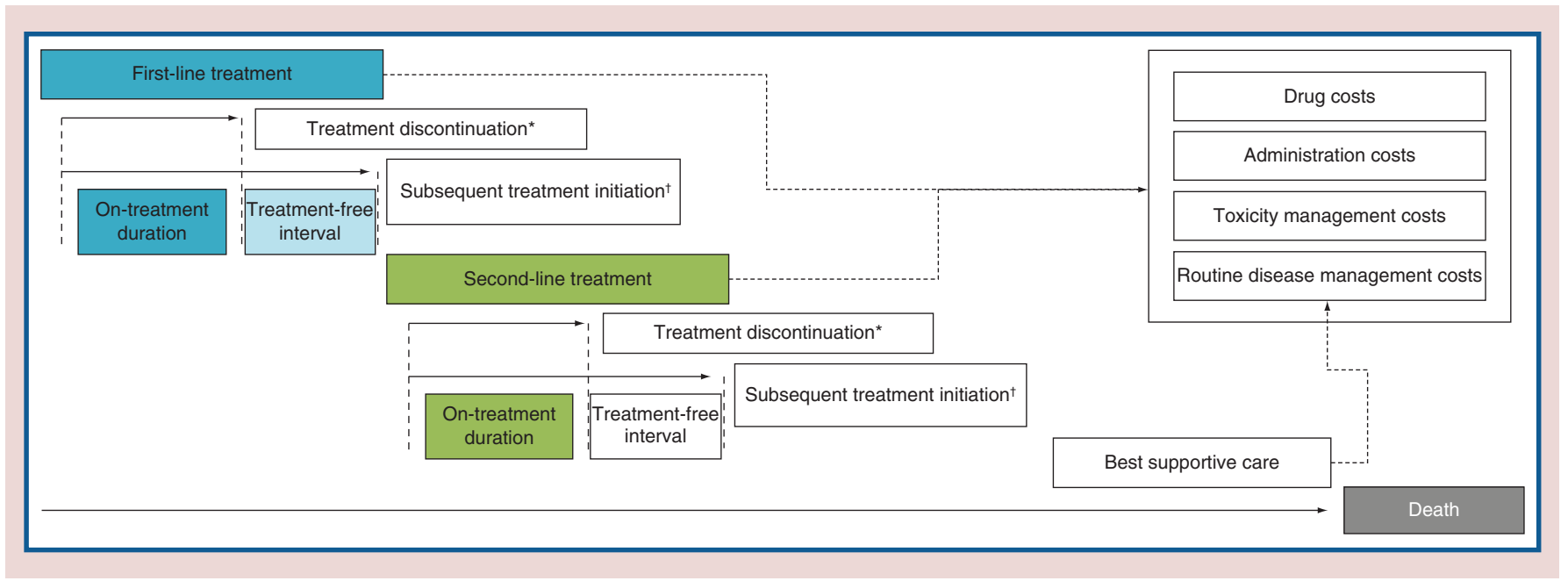

Figure 1. Structure of the simulation model.

*For BRAF + MEK inhibitor starting sequences, progression-free survival was used as proxy for treatment duration.

†Time to subsequent treatment initiation was only available for first-line treatment with anti-PD-1 and anti-PD-1 + anti-CTLA-4 agents. For second-line treatment, published data were not available for time to subsequent treatment initiation. CTLA-4: Cytotoxic T lymphocyte antigen-4; PD-1: Programmed death 1.

ipilimumab for anti-PD-1 + anti-CTLA-4, nivolumab and pembrolizumab for anti-PD-1 (calculated as an average of both) and dabrafenib plus trametinib for BRAF + MEK inhibitors (Supplementary Table 1).

\section{Model structure}

For each treatment sequence evaluated, the model simulated a cohort of patients with a unique set of baseline characteristics. Based on the patient characteristics from the CheckMate 067 and 069 trials, the model predicted the time to clinical events for each line of treatment included in a sequence (Figure 1).

At model initiation, patients start with a first-line treatment and may discontinue treatment for any reason. Upon discontinuation, patients may remain treatment free for any length of time before initiating a subsequent second-line therapy. Patients whose disease progresses while on a first-line treatment or during the treatment-free interval may initiate a second-line treatment. Because of the lack of available data, the model did not include a treatment-free interval after the second-line treatment. In the event of disease progression on a second-line treatment, patients may move onto best supportive care. Death may occur at any time in the model. The costs accrued during treatment include those associated with drug acquisition, administration and adverse event management, as well as disease management costs over patients' lifetimes. Quality of life was also considered, with disutilities due to adverse events based on the time to resolution of the specific adverse event.

Statistical analyses and efficacy modeling have been previously described [26]. Additional details of efficacy modeling are provided in the Supplementary Material.

\section{Modeling of treatment sequences initiated with immuno-oncology agents}

As previously described [26], data for nivolumab alone and in combination with ipilimumab were extrapolated from the CheckMate 067 and CheckMate 069 trials. The efficacy of first-line pembrolizumab was considered to be equivalent to that of nivolumab, based on clinical opinion and supported by efficacy data from network meta-analyses $[38,39]$ and similar median treatment duration for pembrolizumab and nivolumab observed in clinical trials $[4,8]$. A set of sequential risk equations were derived using pooled patient-level data from 891 patients with advanced melanoma included in the CheckMate 067 and CheckMate 069 trials. The sample size was sufficient to establish the impact of individual patient characteristics, including the presence of $B R A F$ mutation, and interim disease milestones (i.e., treatment duration or treatment-free interval) on long-term outcomes such as overall survival. For each patient in the model, the risk equations predicted time on first-line treatment, time to subsequent treatment and time on second-line treatment. The competing risk of death was estimated for each phase in the treatment sequences. The presence of a $B R A F$ mutation was retained as an important covariate in equations for time 


\begin{tabular}{|c|c|c|c|c|}
\hline & \multicolumn{2}{|c|}{ Anti-PD-1 } & \multirow{2}{*}{$\begin{array}{l}\text { Anti-PD-1 + anti-CTLA-4 } \\
\text { Nivolumab + ipilimumab }\end{array}$} & \multirow{2}{*}{$\begin{array}{l}\text { BRAFi + MEKi } \\
\text { Dabrafenib + trametinib }\end{array}$} \\
\hline & Nivolumab & Pembrolizumab & & \\
\hline Drug cost & $\$ 13,280$ & $\$ 13,083$ & $\begin{array}{l}\text { Induction: } \$ 54,152^{\dagger} \\
\text { Maintenance: } \$ 13,280\end{array}$ & $\$ 20,423$ \\
\hline Administration cost & $\$ 456$ & $\$ 304$ & $\begin{array}{l}\text { Induction: } \$ 667^{\dagger} \\
\text { Maintenance: } \$ 456\end{array}$ & $\$ 0$ \\
\hline \multicolumn{5}{|c|}{ Grade $3 / 4$ adverse event management cost } \\
\hline First line & $\$ 36$ & $\$ 30$ & $\$ 414$ & $\$ 25$ \\
\hline Second line & $\$ 4$ & $\$ 7$ & - & $\$ 96$ \\
\hline \multicolumn{5}{|c|}{ Grade $3 / 4$ immune-related adverse event management cost } \\
\hline First line & $\$ 26$ & $\$ 26$ & $\$ 170$ & - \\
\hline Second line & $\$ 0$ & $\$ 0$ & - & - \\
\hline \multicolumn{5}{|l|}{ Disease management cost: first line } \\
\hline On treatment, progression free & $\$ 482$ & $\$ 482$ & $\$ 798$ & $\$ 537$ \\
\hline On treatment, progressed & $\$ 1176$ & $\$ 1176$ & $\$ 1230$ & $\$ 537$ \\
\hline Off treatment, progression free $\ddagger$ & $\$ 188$ & $\$ 188$ & $\$ 263$ & $\$ 843$ \\
\hline Off treatment, progressed $\ddagger$ & $\$ 1608$ & $\$ 1608$ & $\$ 1298$ & $\$ 843$ \\
\hline \multicolumn{5}{|c|}{ Disease management cost: second line } \\
\hline On treatment & $\$ 395$ & $\$ 395$ & - & $\$ 537$ \\
\hline Off treatment & $\$ 688$ & $\$ 688$ & - & $\$ 843$ \\
\hline \multicolumn{5}{|c|}{$\begin{array}{l}{ }^{\dagger} \text { Induction costs were applied for four doses, after which nivolumab maintenance costs were considered. } \\
\text { † Hospitalization and surgery costs in the off-treatment phase for immuno-oncology therapies were capped after } 28 \text { months based on clinical opinion. All other costs were continued } \\
\text { beyond } 28 \text { months. } \\
\text { BRAFi: BRAF inhibitor; CTLA-4: Cytotoxic T-lymphocyte antigen 4; MEKi: MEK inhibitor; PD-1: Programmed death } 1 .\end{array}$} \\
\hline
\end{tabular}

to second-line treatment initiation, survival in the treatment-free interval and survival on second-line treatment. The predictive validity of the risk equations was established by comparing the model-simulated outcomes versus the observed CheckMate 067 and CheckMate 069 trial data. Detailed derivations, implementation of risk equations and the comparison of fitted and original Kaplan-Meier curves are presented in Supplementary Tables 2 \& Supplementary Figures 1-4. The duration of progression-free survival on second- and third-line treatment was based on parametric distributions fitted to the Kaplan-Meier progression-free curves reported in second-line clinical trials [3,5,40-42]. The parameters for modeling progression are presented in Supplementary Table 3.

\section{Modeling of treatment sequences initiated with targeted therapy (BRAF + MEK inhibitors)}

In the absence of head-to-head clinical trial data between immuno-oncology agents and BRAF + MEK inhibitors and given the lack of common reference arms in these studies $[13,14,18,23,24,33]$, a matching adjusted indirect comparison was conducted. This analysis estimated the treatment effect of dabrafenib plus trametinib (COMBI trials) compared with nivolumab plus ipilimumab (CheckMate trials) by adjusting for differences in patient characteristics. Patients on BRAF + MEK inhibitor therapy were assumed to immediately initiate second-line treatment, based on a median time to subsequent treatment of 12 days reported in the COMBI-d trial [24].

The hazard ratios (HRs) between dabrafenib plus trametinib and nivolumab plus ipilimumab for overall survival and progression-free survival (used as a proxy for treatment duration of BRAF + MEK inhibitor combinations) were estimated using the matching-adjusted comparison [34]. There was evidence of nonproportionality between the HRs for targeted versus immuno-oncology therapy for both clinical outcomes. For overall survival, the hazard of death for nivolumab plus ipilimumab slowed considerably over time, showing superiority after 12 months. To model this, a separate HR was applied before and after 12 months. Similarly, for treatment duration, separate time-dependent HRs were applied for time points 0-5 months, 5-12 months and after 12 months. HRs are presented in Supplementary Table 4.

\section{Costs}

The drug administration, disease management and adverse event costs were estimated from USA's third-party payer perspective over a patient lifetime time horizon (30 years). Monthly cost inputs are shown in Table 1 . The drug and administration costs per month were estimated using the drug acquisition cost, route of administration, unit costs 
for administration (payer reimbursement for intravenous drug administration in physician's office and hospital outpatient settings), recommended dose and dosing frequency based on publicly available sources (i.e., RedBook and Medicare Payment limits), prescribing information and clinical trials (Supplementary Tables 1 \& 5-8) [40,41,4349]. The model considered grade $3 / 4$ treatment-related and immune-related adverse events reported in clinical trials (Supplementary Tables $1 \&$ 9-11). Inclusion of adverse events were limited to those of grade $3 / 4$ due to their economic impact. Although adverse events of lower grades may occur frequently, their costs and impact on quality of life were not considered in the model. Grade 5 adverse events were excluded due to their very low incidence. Adverse event management costs were obtained from the published literature [27,50]. To address data gaps, adverse events were assumed to be treated in the inpatient setting, and the cost was obtained from the Healthcare Cost and Utilization Project National Inpatient Sample database (Supplementary Tables 12 \& 13) [51]. To determine routine disease management costs, a statistical analysis was conducted to understand the resource use patterns from the CheckMate 067 and CheckMate 069 analysis. The rates of resource use (concomitant medications, hospitalizations, laboratory tests, procedures, surgeries and consultation) were analyzed by treatment status (first-line on-treatment, first-line off-treatment, second-line on-treatment, second-line off-treatment), disease status (progression free, progressed) and treatment arm (Supplementary Tables 14-19). Resource item unit costs were obtained from published sources and drug costs were based on published wholesale acquisition costs [47,48,51]. Unit costs were inflated using the medical consumer price index from the USA if they were not in 2016 US dollars.

\section{Quality of life}

The model considered utility values for progression-free (0.79) and progressed health states (0.75), estimated from responses to the EuroQoL-5 Dimensions in the CheckMate 067 trial. The utility index scores were estimated using the UK time trade-off method [52]. It is well known that different methods to derive health state utilities can result in large differences in the estimates [53]; however, in the absence of trial-based adverse-event disutility data, we believe that our approach was conservative.

Adverse-event-related disutilities were considered depending on the setting of care, and the incidence was obtained from clinical trials (outpatient, -0.13 ; inpatient, -0.17) [54]. The duration of disutility related to adverse events was based on the time to resolution of events reported in CheckMate 067. For adverse events not reported in CheckMate 067, the observed length of stay from the Healthcare Cost and Utilization Project National Inpatient Sample database was used. Supplementary Table 20 shows the adverse event and immune-related adverse event disutilities per year.

\section{Analyses}

Point estimates for total life-years, quality-adjusted life-years and lifetime costs by sequence, as well as incremental costs, quality-adjusted life-years relative to most often used current sequences and their ratios, are presented. Health outcomes and costs are reported as discounted, using an annual discount rate of $3.0 \%$. A probabilistic analysis was conducted to estimate the impact of parameter uncertainty on results. The analysis inputs were varied per the standard guidelines by the International Society for Pharmacoeconomics and Outcomes Research - Society for Medical Decision Making task force [55]. Efficacy risk equations used a variance-covariance matrix. Cost inputs assumed gamma distribution, and standard error was assumed to be $20 \%$ of the mean. Quality-of-life inputs used beta distribution, and standard error was assumed to be $10 \%$ of the mean.

\section{Results}

Based on the observed CheckMate trial data, the model generated results beyond the clinical trial follow-up period, which were extrapolated over the patient's lifetime.

\section{Health outcomes \& cost}

Treatment with first-line anti-PD-1 + anti-CTLA-4 followed by second-line BRAF + MEK inhibitors was associated with the longest survival gain in total life-years. Total life-years were approximately 5 years higher when initiated with first-line anti-PD-1 + anti-CTLA-4 (8.4 years) compared with first-line BRAF + MEK inhibitors (3.2 years) (Figure 2$)$. The estimated mean treatment-free interval duration was longer for first-line anti-PD-1 + anti-CTLA-4 (4.3 years) compared with first-line anti-PD-1 alone (2.5 years) (Figure 3). Use of BRAF + MEK inhibitors as second-line treatment following immuno-oncology therapy (anti-PD-1 or anti-PD-1 + anti-CTLA- 


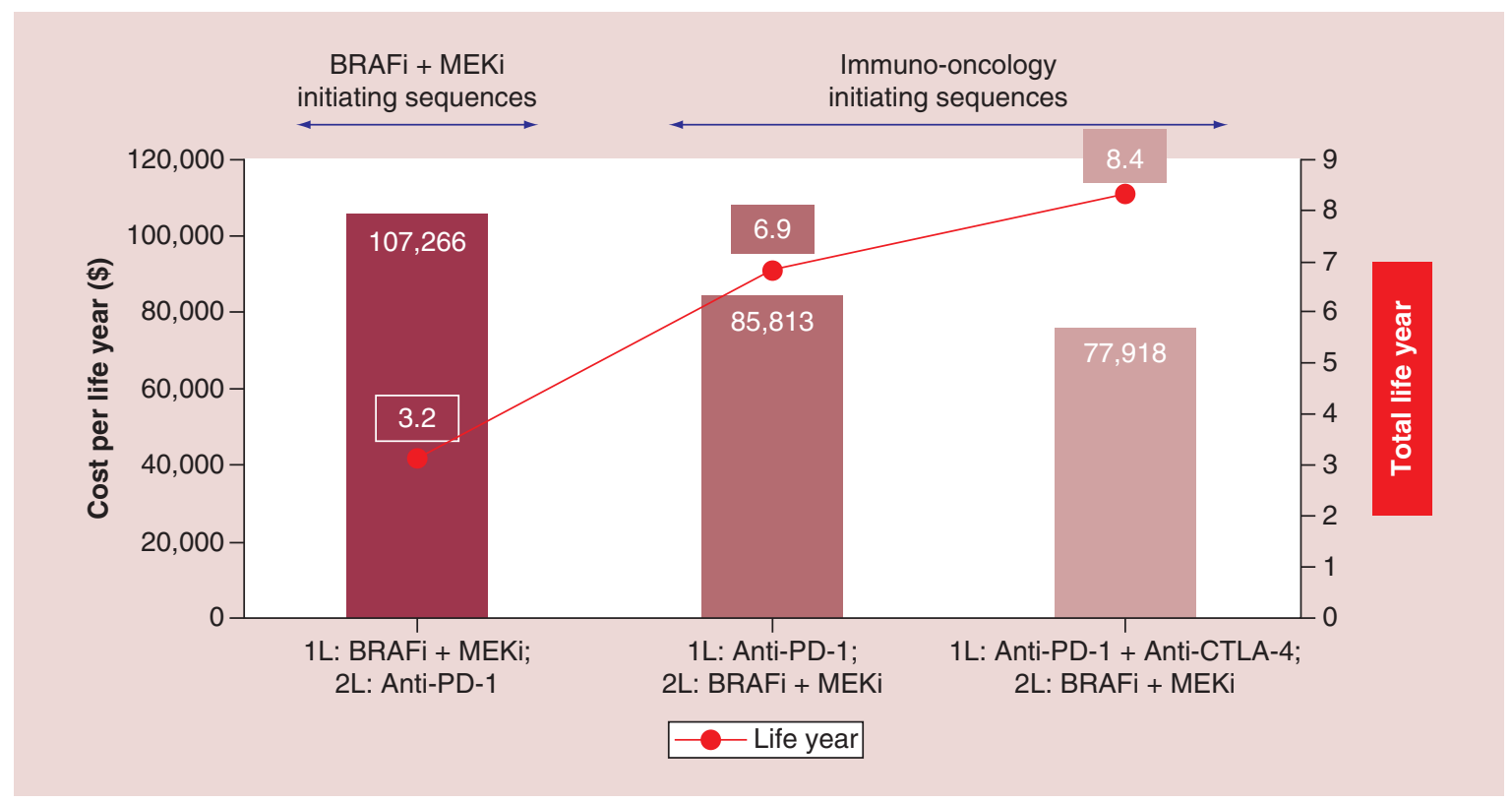

Figure 2. Cost and health outcomes.

1L: First-line; 2L: Second-line; Anti-PD-1: Nivolumab/pembrolizumab; Anti-PD-1 + anti-CTLA-4: Nivolumab + ipilimumab; BRAFi: BRAF inhibitor; BRAFi + MEKi: Dabrafenib + trametinib; CTLA-4: Cytotoxic T lymphocyte antigen-4; MEKi: MEK inhibitor; PD-1: Programmed death 1.

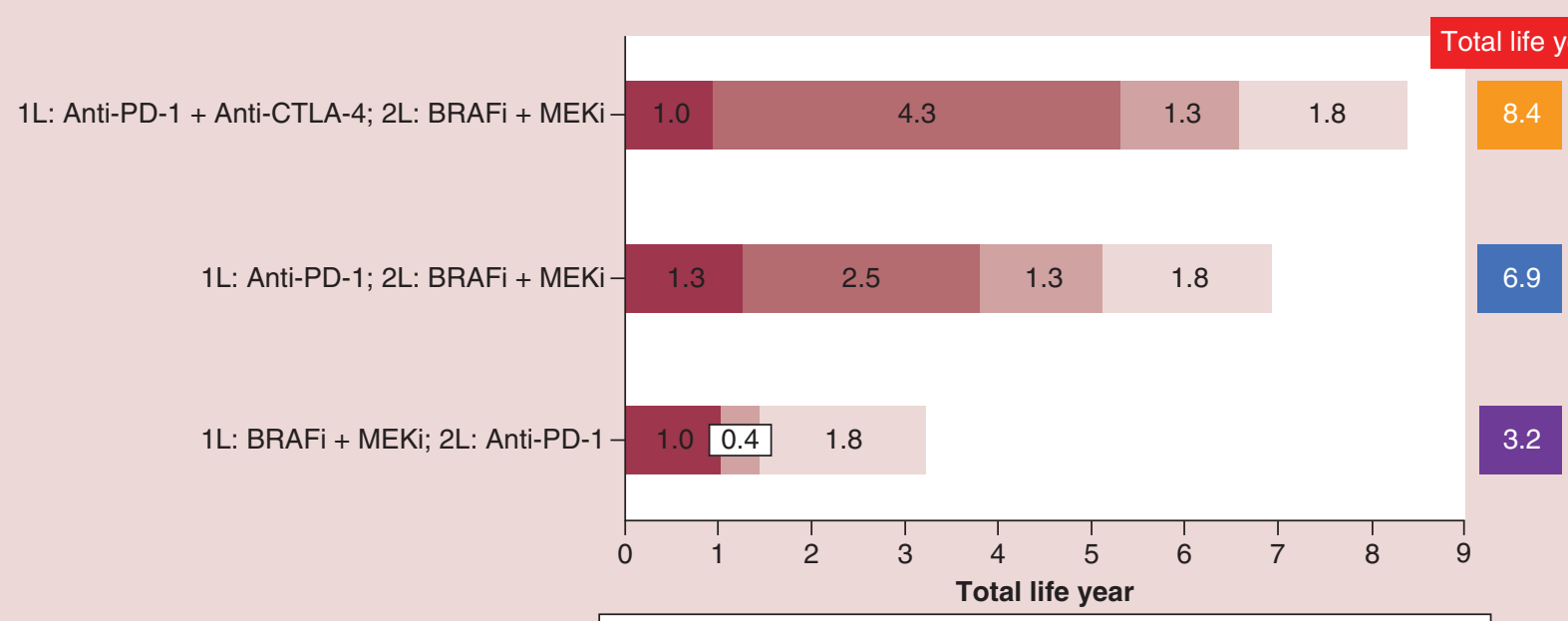

1L: on treatment $\square$ 1L: treatment-free $\square 2 \mathrm{~L}$ : on treatment $\square$ Post $2 \mathrm{~L}$

Figure 3. Total life-years on treatment sequences.

1L: First-line; 2L: Second-line; Anti-PD-1: Nivolumab/pembrolizumab; Anti-PD-1 + anti-CTLA-4: Nivolumab + ipilimumab; BRAFi: BRAF inhibitor; BRAFi + MEKi: Dabrafenib + trametinib; CTLA-4: Cytotoxic T lymphocyte antigen-4; MEKi: MEK inhibitor; PD-1: Programmed death 1.

4) provided numerically higher life-year benefit as with first-line BRAF + MEK inhibitors (1.3 vs 1.1 years, respectively).

Treatment with anti-PD-1 + anti-CTLA- 4 followed by subsequent BRAF + MEK inhibitors was also associated with the longest gain in total quality-adjusted life-years: 6.5 years with first-line anti-PD-1 + anti-CTLA-4, 5.4 years with first-line anti-PD-1, and 2.6 years with first-line BRAF + MEK inhibitors. 


\begin{tabular}{|c|c|c|c|}
\hline & $\begin{array}{l}\text { ICER vs } \\
\text { 1L: BRAFi + MEKi } \\
\text { 2L: Anti-PD-1 }\end{array}$ & $\begin{array}{l}\text { ICER vs } \\
\text { 1L: Anti-PD-1 + Anti-CTLA-4 } \\
\text { 2L: BRAFi + MEKi }\end{array}$ & $\begin{array}{l}\text { ICER vs } \\
\text { 1L: Anti-PD-1 } \\
\text { 2L: BRAFi + MEKi }\end{array}$ \\
\hline $\begin{array}{l}\text { 1L: BRAFi + MEKi } \\
\text { 2L: Anti-PD-1 }\end{array}$ & - & $\$ 79,124^{\dagger}$ & $\$ 89,067^{\dagger}$ \\
\hline $\begin{array}{l}\text { 1L: Anti-PD-1 + anti-CTLA-4 } \\
\text { 2L: BRAFi + MEKi }\end{array}$ & $\$ 79,124 \ddagger$ & - & $\$ 54,273^{\ddagger}$ \\
\hline $\begin{array}{l}\text { 1L: Anti-PD-1 } \\
\text { 2L: BRAFi + MEKi }\end{array}$ & $\$ 89,067 \ddagger$ & $\$ 54,273^{\dagger}$ & - \\
\hline \multicolumn{4}{|c|}{$\begin{array}{l}\text { †Less effective and less costly. } \\
\text { ¥ More effective and more costly. } \\
\text { 1L: First line; 2L: Second line; BRAFi: BRAF inhibitor; CTLA-4: Cytotoxic T lymphocyte antigen-4; ICER: Incremental cost-effectiveness ratio; MEKi: MEK inhibitor; PD-1: Programmed death } \\
\text { 1. }\end{array}$} \\
\hline
\end{tabular}

The estimated total lifetime costs were highest for the first-line anti-PD-1 + anti-CTLA-4 sequence $(\$ 656,692)$ compared with the first-line anti-PD-1 $(\$ 595,727)$ and first-line BRAF + MEK inhibitor $(\$ 345,693)$ sequences. In this regard, the cost of first-line treatment was the largest contributor of total lifetime costs for sequences initiating with BRAF + MEK inhibitors $(\$ 263,165)$. For sequences initiating with immuno-oncology agents, costs of second-line treatment (BRAF + MEK inhibitor) were the largest contributor of total lifetime costs (anti-PD-1 + anti-CTLA-4 initiating sequences, $\$ 324,994$; anti-PD-1 initiating sequences, $\$ 333,304$ ).

However, the average cost per life-year was lowest for the first-line anti-PD-1 + anti-CTLA-4 sequence $(\$ 77,918)$ compared with the first-line anti-PD-1 $(\$ 85,813)$ and first-line BRAF + MEK inhibitor $(\$ 107,266)$ sequences. Similarly, the average cost per quality-adjusted life-year was also lowest for the first-line anti-PD-1 + anti-CTLA4 sequence $(\$ 101,276)$ compared with first-line anti-PD-1 $(\$ 111,124)$ and first-line BRAF + MEK inhibitor $(\$ 135,372)$ sequences.

\section{Incremental cost-effectiveness ratio}

Incremental costs, incremental quality-adjusted life-years and the calculated incremental cost-effectiveness ratios (ICERs) for treatment sequences are presented in Table 2. Compared with the first-line BRAF + MEK inhibitor sequence, the ICERs of first-line anti-PD-1 + anti-CTLA- 4 sequence and first-line anti-PD-1 sequence were $\$ 79,124$ and $\$ 89,067$ per quality-adjusted life-years, respectively. The ICER of first-line anti-PD-1 + anti-CTLA4 sequence versus first-line anti-PD-1 sequence was US $\$ 54,273$ per quality-adjusted life-year. All ICERs were within the willingness-to-pay threshold of US $\$ 150,000$.

\section{Scenario analyses}

Scenario analyses were conducted to determine the impact of a maximum treatment duration of first-line immunooncology treatments (24 months) on model outcomes. The results of the scenario analyses are shown in Supplementary Table 21. A maximum first-line treatment duration of 2 years with immuno-oncology therapy provided a similar survival gain and reduced the cost of immuno-oncology initiating sequences by approximately $\$ 53,000-\$ 67,000$ with approximately $0.1-0.2$ life-years lost.

\section{Probabilistic sensitivity analysis}

The cost-effectiveness acceptability curve in Figure 4 shows that for up to a willingness-to-pay value of $\$ 80,000$ per quality-adjusted life-year, a first-line BRAF + MEK inhibitor followed by an anti-PD-1 was the most likely costeffective treatment option. At higher willingness-to-pay values, first-line anti-PD-1 + anti-CTLA- 4 followed by second-line BRAF + MEK inhibitors was the most likely cost-effective option with a probability of approximately 40-90\%. For a first-line anti-PD-1 with a second-line BRAF + MEK inhibitor sequence, the probability of costeffectiveness increased to $28 \%$ at a willingness-to-pay value of $\$ 112,500$ and decreased thereafter to approximately $10 \%$.

\section{One-way sensitivity analyses}

One-way sensitivity analyses were conducted on the model inputs that had the greatest impact on life-years, quality-adjusted life-years and lifetime costs on treatment sequences (Supplementary Table 22). Model results were sensitive to the first- and second-line treatment effects in the risk equations derived from the CheckMate trials 


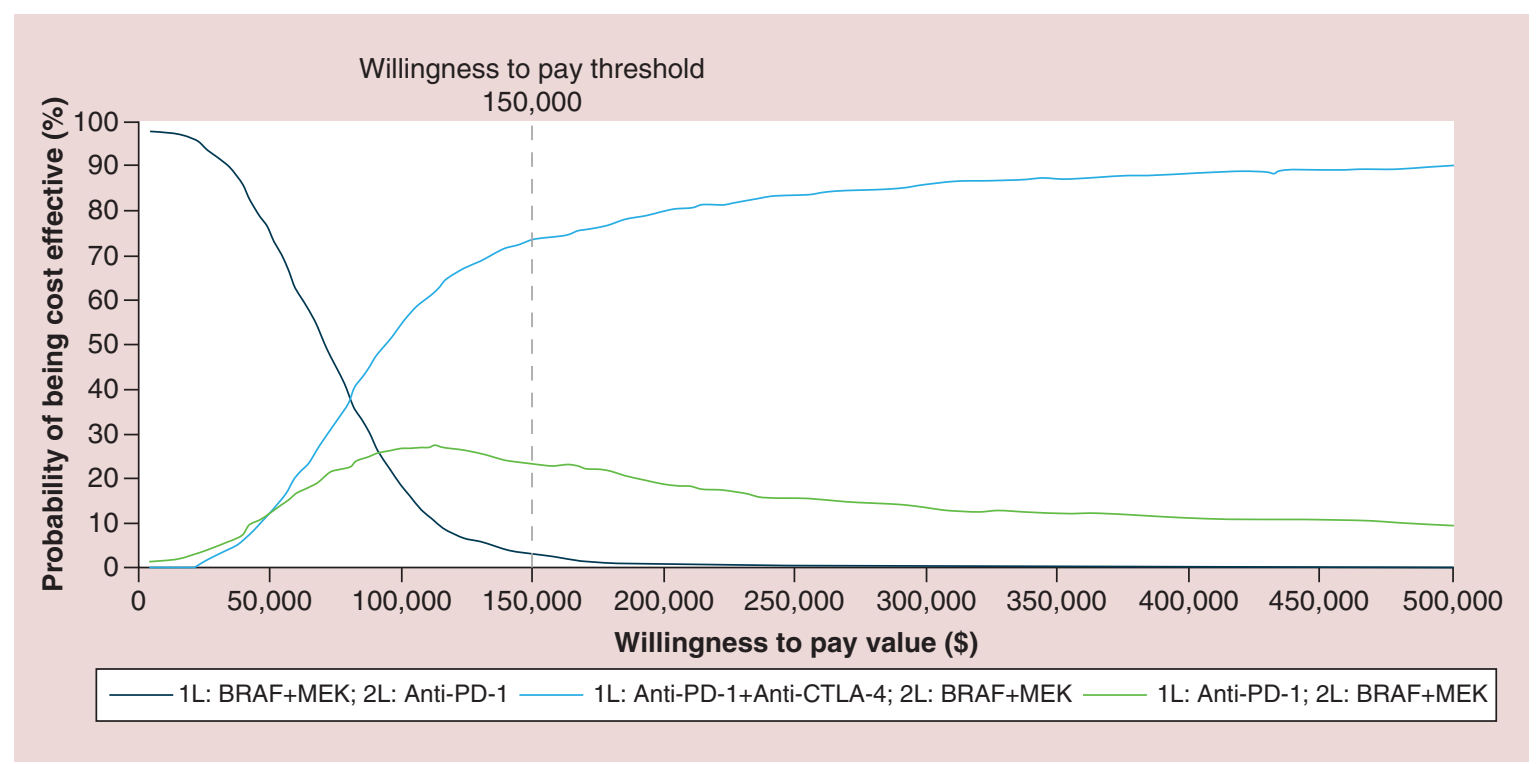

Figure 4. Cost-effectiveness acceptability curve - quality-adjusted life-years.

1L: First-line; 2L: Second-line; CTLA-4: Cytotoxic T lymphocyte antigen-4; PD-1: Programmed death 1.

on first-line anti-PD-1 sequence and to the overall survival HRs on first-line BRAF + MEK inhibitor sequence. Outcomes of anti-PD-1 + anti-CTLA-4 initiating sequence were sensitive to variations in the time to subsequent treatment. Quality-adjusted life-years were also impacted by utility values for the post-progression health state. In terms of cost inputs, drug costs had the largest impact on lifetime cost results. Additional results of univariate sensitivity analyses are available in the Supplementary Material.

\section{Discussion}

Similar to the treatment landscape in $B R A F$ wild-type advanced melanoma, treatment options for patients with $B R A F$-mutant melanoma have evolved considerably in recent years. The treatment paradigm has shifted from chemotherapy-based and cytokine-based therapy to $B R A F$-targeted and immune-checkpoint blockade approaches. Although the newer treatments have demonstrated improved response and superior overall survival for patients with $B R A F$-mutant advanced melanoma, most patients receive multiple lines of treatment due to disease progression or toxicity, thus posing a challenge to clinical decision making in terms of the optimal sequence of the available treatment agents. Consistent with our earlier report [26], in this study we have estimated the optimal positioning and benefit of the anti-PD-1 + anti-CTLA- 4 combination compared with other therapies and quantified the overall economic burden associated with multiple lines of sequential treatments in patients with $B R A F$-mutant melanoma. While ongoing clinical trials investigate the optimal sequence of immuno-oncology and targeted agents [25,56], in the absence of head-to-head data, a simulation model such as the one presented in this study can shed light on expected clinical outcomes and economic consequences of various first- and second-line treatment sequences. To date, we were not able to find published economic analyses in patients with $B R A F$-mutant melanoma against which we could compare our results; however, a few cost-effectiveness studies are available in $B R A F$ wild-type patients [26,57].

The average cost per life-year was lowest for immuno-oncology initiating sequences $(\$ 78,000-\$ 86,000)$ compared with BRAF + MEK inhibitor initiating sequences $(\$ 107,000)$, despite higher lifetime costs. This was due in large part to the fact that patients treated with immuno-oncology therapies, particularly the anti-PD-1 + antiCTLA-4 combination, often experience durable clinical benefit despite cessation of treatment, which can delay or even eliminate the need for subsequent treatment [19]. Such a treatment-free period is often only implicitly included in cost-effectiveness models of advanced melanoma using survival partition models or Markov models [57].

Our findings showed that, of the treatment sequences evaluated, first-line anti-PD-1 + anti-CTLA- 4 followed by the BRAF + MEK inhibitor sequence was associated with the highest overall survival - approximately an additional 
5 years of mean survival over the BRAF + MEK inhibitor initiating sequence. This was driven primarily by the extended treatment-free interval following the first-line treatment with anti-PD-1 + anti-CTLA-4.

The longer overall survival was associated with higher lifetime total costs $(\$ 656,692)$, but the ICER per qualityadjusted life-year for the first-line immuno-oncology combination versus the first-line anti-PD-1 $(\$ 54,273)$ and the ICER per quality-adjusted life-year for the first-line immuno-oncology combination versus first-line BRAF + MEK inhibitors $(\$ 79,124)$ were within the willingness-to-pay threshold in the USA [58]. The primary contributors of costs in the model were the drug costs associated with combination drug therapies (i.e., anti-PD-1 + anti-CTLA-4 and BRAF + MEK inhibitor).

Notably, the model estimated a longer treatment duration for the BRAF + MEK inhibitor combination when used as a second-line treatment following immuno-oncology therapy than when used as a first-line treatment. This suggests that immuno-oncology therapies may continue to provide prolonged benefit even after treatment discontinuation or, although the mechanisms remain unclear, may have the ability to prime patients for the use of BRAF + MEK inhibitors as subsequent therapy. It is also possible that the use of different data sources for time on treatment with first-line and second-line BRAF + MEK inhibitors may have introduced bias (COMBI trials for first-line; pooled CheckMate trials for second-line). In contrast to our findings, a recent, small, real-world study, albeit not statistically controlled for differences in patient characteristics, reported that BRAF inhibition was less effective as salvage therapy for 22 patients who failed first-line anti-PD-1 therapy compared with frontline treatment [59]. It is possible, that patients resistant to PD-1 inhibitors may be cross-resistant to BRAF inhibitors as well.

There are several features that can be argued as strengths for this study. The use of a matching-adjusted-indirect comparison enabled comparison of clinical outcomes across the COMBI and CheckMate trials, adjusting for differences in patient characteristics and for treatment-effect HRs that change over time (rather than assuming constant HRs). The integration of a treatment-free interval, a novel outcome of immuno-oncology-initiating sequences, is another strength, as extended drug-free periods while maintaining disease control can have a significant impact on cost-effectiveness. Use of an extensive list of adverse events that was captured and validated through expert clinical opinion is another strength. Disutility due to adverse events was calculated based on the reported time to resolution of the adverse events, which can be considered as closely matching patient experience.

This study has limitations. Due to the unavailability of long-term follow-up in the included studies, modeling was used to extrapolate outcomes over patients' lifetimes, which would require a higher level of evidence validation in the future. Some of the model equations (i.e., time on second-line treatment and the risk of death during and after second-line treatment) was limited by the small sample size of pooled data for nivolumab and the nivolumab plus ipilimumab combination. Data had to be pooled across treatment types or a common shape function had to be assumed across various second-line therapies. Second-line use of anti-PD-1 + anti-CTLA-4 following BRAF + MEK inhibitors was excluded in the model due to limited information on clinical outcomes associated with this sequence. Due to paucity of data, time-on-treatment curves for the dabrafenib + trametinib combination were approximated using the progression-free survival data. In addition, due to the lack of available data, it was not possible to adequately model the benefits of third or subsequent lines of therapy.

Treatment-free periods for BRAF + MEK inhibitor therapies and for second-line treatment with immunooncology agents were not included in the model. For BRAF + MEK inhibitor therapies, the patient-level data necessary to examine treatment-free interval were not available; however, based on 3-year COMBI-d data, the median time to subsequent therapy initiation was 12 days, suggesting that patients do not spend significant time without treatment [23]. Currently, data are not available from completed prospective randomized controlled trials of combination immuno-oncology therapy in patients with $B R A F$-mutant melanoma who failed prior BRAF + MEK inhibitor therapy. Assuming substantial treatment-free interval gains after second-line or third-line immunooncology therapy, the actual total life-years experienced by patients treated with first-line targeted therapy are likely to be much closer to the total life-years of those treated with first-line immunotherapy than the current model estimates. However, patients who fail first-line therapy may or may not be able to make it into subsequent lines of therapy. Therefore, it would be difficult to make conclusions in the absence of clinical trial data that address the likelihood of starting second-line immuno-oncology therapy and the likelihood of responding and surviving. Ongoing studies (EA6134) will be able to address these questions and also validate our model. 


\section{Conclusion}

In this study, initiating first-line treatment with an immuno-oncology agent provided a longer survival benefit compared with initiating treatment with a BRAF + MEK inhibitor combination. These results were driven by a long treatment-free interval and, in many cases, the lack of a need for subsequent therapy. This led to a lower average cost per life-year and cost per quality-adjusted life-year gained for immuno-oncology initiating sequences. Because these data may be confounded by unknown factors that could not be accounted for, these findings will require validation in prospective, randomized, clinical trials such as NCT02224781 [25].

\section{Summary points}

- Multiple checkpoint inhibitors and targeted therapies have been approved in recent years for the treatment of patients with BRAF-mutant advanced melanoma. Thus creating more options in the treatment of this fatal disease, but also leading to difficult treatment decisions.

- A cost-effectiveness analysis was conducted to distinguish between various treatment sequences, initiating with the anti-PD-1 + anti-CTLA-4 combination, anti-PD-1 monotherapy or the BRAF + MEK inhibitor used in treating patients with BRAF-mutant advanced melanoma.

- A patient-level simulation model was developed using a discretely integrated condition event structure to evaluate cost and health outcomes over a patient's lifetime.

- The analysis is based on patient-level data from the Phase III CheckMate 067 and Phase II CheckMate 069 clinical trials and a matching adjusted indirect comparison (using COMBI-v and COMBI-d trials), and takes into account several relationships between patient characteristics and outcomes (treatment duration, treatment-free interval, progression and survival).

- The estimated survival was higher for sequences initiating with anti-PD-1 + anti-CTLA-4 (mean 8.4 years) than for anti-PD-1 monotherapy (mean 6.9 years) or BRAF + MEK inhibitors (mean 3.2 years).

- The incremental cost-effectiveness ratio per quality-adjusted life-year of survival for first-line anti-PD-1 + anti-CTLA-4 combination was $\$ 54,273$ versus first-line anti-PD-1 and $\$ 79,124$ versus first-line BRAF + MEK inhibitor.

- Initiating treatment with anti-PD-1 + anti-CTLA-4 was more cost-effective than initiation with anti-PD-1 monotherapy or BRAF + MEK inhibitors.

Financial \& competing interests disclosure

This study was supported by Bristol-Myers Squibb (NJ, USA). A Tarhini has disclosed a consulting or advisory role with BristolMyers Squibb and having received research funding from Bristol-Myers Squibb, Merck Sharp \& Dohme, Amgen, Novartis, Incyte and Prometheus Laboratories. A Tarhini has also disclosed a consulting or advisory role with Novartis, Genentech-Roche, Array Biopharma, NewLink Genetics, Incyte, HUYA, EMD Serono, Pfizer, Sanofi-Aventis and Regeneron. D McDermott has disclosed a consulting or advisory role with Bristol-Myers Squibb, Merck, Pfizer and Genentech. M Atkins has disclosed a consulting or advisory role with Genentech, Pfizer, Novartis, GlaxoSmithKline, C-Cam, X4 Pharma, Amgen, Lilly, Alkermes, Infinity Pharmaceuticals, Genoptix, Bristol-Myers Squibb, Nektar and Merck and having received honoraria from Merck. MM Regan has disclosed a consulting or advisory role with Merck (individual) and Ipsen (institution) and having received research funding (all to institution) from Veridex, Oncogenex, Pfizer, Ipsen, Novartis, Merck, Ferring, Celgene, AstraZeneca, Pierre Fabre and Bayer. A Benedict, A Ambavane and $\vee$ Aponte-Ribero serve as paid consultants for Bristol-Myers Squibb. K Gupte-Singh, C Ritchings and S Rao are employees of Bristol-Myers Squibb. The authors have no other relevant affiliations or financial involvement with any organization or entity with a financial interest in or financial conflict with the subject matter or materials discussed in the manuscript apart from those disclosed.

The authors thank K Poinsett-Holmes (Evidera, MD, USA) for medical writing/editorial assistance during the development of the manuscript. Editorial assistance was provided by K Parai and C Hunsberger at StemScientific, an Ashfield Company, funded by Bristol-Myers Squibb.

\section{Acknowledgments}

The authors would like to thank the patients and families who made the trials possible and the clinical study teams who participated in the trials. Study design, data analysis and interpretation and review of manuscript occurred in collaboration with Bristol-Myers Squibb and non-Bristol-Myers Squibb authors. 


\section{Author contributions}

Study conceptualization was done by A Tarhini, A Benedict, A Ambavane and S Rao. A Benedict and A Ambavane worked on the methodology. $V$ Aponte-Ribero worked on the formal analysis. Investigation, writing of original draft, review and editing were done by all authors.

\section{Data sharing}

BMS policy on data sharing may be found at www.bms.com/researchers-and-partners/independent-research/data-sharing-reque st-process.html.

\section{Open access}

This work is licensed under the Attribution-NonCommercial-NoDerivatives 4.0 Unported License. To view a copy of this license, visit http://creativecommons.org/licenses/by-nc-nd/4.0/

\section{Supplementary material}

To view the supplementary material that accompany this paper please visit the journal website at: www.futuremedicine.com/doi/s uppl/10.2217/imt-2018-0168

\section{References}

Papers of special note have been highlighted as: $\bullet$ of interest; $\bullet \bullet$ of considerable interest

1. National Cancer Institute. Surveillance, epidemiology, and end results program. SEER Cancer Stat Facts: Melanoma of the Skin 2018. https://seer. cancer.gov/statfacts/html/melan.html

2. Jang S, Atkins MB. Which drug, and when, for patients with BRAF-mutant melanoma? Lancet Oncol. 14(2), e60-e69 (2013).

3. Hodi FS, O’Day SJ, McDermott DF et al. Improved survival with ipilimumab in patients with metastatic melanoma. N. Engl. J. Med. 363(8), 711-723 (2010).

4. Robert C, Schachter J, Long GV et al. Pembrolizumab versus ipilimumab in advanced melanoma. N. Engl. J. Med. 372(26), 2521-2532 (2015).

5. Ribas A, Puzanov I, Dummer R et al. Pembrolizumab versus investigator-choice chemotherapy for ipilimumab-refractory melanoma (KEYNOTE-002): a randomised, controlled, Phase 2 trial. Lancet Oncol. 16(8), 908-918 (2015).

- Findings from this randomized Phase II trial established pembrolizumab as a new standard of care for the treatment of ipilimumab-refractory melanoma.

6. Robert C, Long GV, Brady B et al. Nivolumab in previously untreated melanoma without BRAF mutation. N. Engl. J. Med. 372(4), 320-330 (2015).

7. Weber JS, D’Angelo SP, Minor D et al. Nivolumab versus chemotherapy in patients with advanced melanoma who progressed after anti-CTLA-4 treatment (CheckMate 037): a randomised, controlled, open-label, phase 3 trial. Lancet Oncol. 16(4), 375-384 (2015).

8. Larkin J, Chiarion-Sileni V, Gonzalez R et al. Combined nivolumab and ipilimumab or monotherapy in untreated melanoma. N. Engl. J. Med. 373(1), 23-34 (2015).

9. Carlino MS, Long GV. Ipilimumab combined with nivolumab: a standard of care for the treatment of advanced melanoma? Clin. Cancer Res. 22(16), 399-328 (2016).

10. Chapman $\mathrm{PB}$, Hauschild A, Robert $\mathrm{C}$ et al. Improved survival with vemurafenib in melanoma with BRAF V600E mutation. N. Engl. J. Med. 364(26), 2507-2516 (2011).

11. Hauschild A, Grob JJ, Demidov LV et al. Dabrafenib in BRAF-mutated metastatic melanoma: a multicentre, open-label, phase 3 randomised controlled trial. Lancet 380(9839), 358-365 (2012).

12. Larkin J, Ascierto PA, Dréno B et al. Combined vemurafenib and cobimetinib in BRAF-mutated melanoma. N. Engl. J. Med. 371(20), 1867-1876 (2014).

13. Robert C, Karaszewska B, Schachter J et al. Improved overall survival in melanoma with combined dabrafenib and trametinib. N. Engl. J. Med. 372(1), 30-39 (2015).

14. Long G, Stroyakovskiy D, Gogas H et al. Dabrafenib and trametinib versus dabrafenib and placebo for Val600 BRAF-mutant melanoma: a multicentre, double-blind, Phase III randomised controlled trial. Lancet 386(9992), 444-451 (2015).

15. Awad MM, Sullivan RJ. Dabrafenib in combination with trametinib for the treatment of metastatic melanoma. Expert Rev. Clin. Pharmacol. 8(1), 25-33 (2015).

16. Dummer R, Ascierto PA, Gogas HJ et al. Encorafenib plus binimetinib versus vemurafenib or encorafenib in patients with BRAF-mutant melanoma (COLUMBUS): a multicentre, open-label, randomised phase 3 trial. Lancet Oncol. 19(5), 603-615 (2018).

17. Medina PJ, Adams VR. PD-1 pathway inhibitors: immune-oncology agents for restoring antitumor immune responses. Pharmacotherapy 36(3), 317-334 (2016). 
18. Hodi FS, Chiarion-Sileni V, Gonzalez R et al. Nivolumab plus ipilimumab or nivolumab alone versus ipilimumab alone in advanced melanoma (CheckMate 067): 4-year outcomes of a multicentre, Phase 3 trial. Lancet Oncol19(11), 1480-1492 (2018).

-• The Phase III CheckMate 067 study demonstrated, that among patients with advanced melanoma, significantly longer overall survival occurred with combination therapy with nivolumab plus ipilimumab or with nivolumab alone than with ipilimumab alone.

19. Postow M, Larkin J, Wolchok JD et al. Pooled 3-year overall survival data from Phase II and Phase III trials of nivolumab (NIVO) combined with ipilimumab (IPI) in advanced melanoma. Presented at: Society for Immunotherapy of Cancer Annual Meeting. MD, USA, 10-12 November 2017.

- Pooled data demonstrated that the nivolumab plus ipilimumab combination resulted in a longer treatment-free interval than nivolumab or ipilimumab alone and that combination treatment led to a higher percentage of patients alive and treatment free at 3 years compared with the monotherapies.

20. Tarhini A, McDermott D, Benedict A et al. Quantifying the treatment-free interval after first-line immuno-oncology (IO) treatment of advanced melanoma. Presented at: 9th World Congress of Melanoma and the 14th International Congress of the Society for Melanoma Research. Brisbane, Australia, 18-21 October 2017.

21. Menzies AM, Long GV. Systemic treatment for BRAF-mutant melanoma: where do we go next? Lancet Oncol. 15(9), e371-e381 (2014).

22. Flaherty KT, Davies MA, Grob JJ, Long GV, Nathan PD, Ribas A. Genomic analysis and 3-year efficacy and safety update of COMBI-d: a phase 3 study of dabrafenib plus trametinib vs dabrafenib monotherapy in patients with unresectable or metastatic BRAF V600E/K-mutant cutaneous melanoma. J. Clin. Oncol. 34(Suppl. 15), 9502 (2016).

23. Long GV, Flaherty KT, Stroyakovskiy D et al. Dabrafenib plus trametinib versus dabrafenib monotherapy in patients with metastatic BRAF V600E/K-mutant melanoma: long-term survival and safety analysis of a phase 3 study. Ann. Oncol. 28(7), 1631-1639 (2017).

- The Phase III COMBI-d study demonstrated that durable survival of greater than 3 years is achievable with dabrafenib + trametinib in patients with $B R A F$ V600-mutant metastatic melanoma.

24. Robert C, Karaszewska B, Schachter J et al. Three-year estimate of overall survival in COMBI-v, a randomized phase 3 study evaluating first-line dabrafenib (D) + trametinib (T) in patients (pts) with unresectable or metastatic BRAF V600E/K-mutant cutaneous melanoma. Ann. Oncol. 27(Suppl. 6), LBA40 (2016).

25. ClinicalTrials.gov. Dabrafenib and trametinib followed by ipilimumab and nivolumab or ipilimumab and nivolumab followed by dabrafenib and trametinib in treating patients with stage III-IV BRAFV600 melanoma (2014). www.clinicaltrials.gov/ct2/show/NCT02224781

26. Tarhini A, Benedict A, McDermott D et al. Sequential treatment approaches in the management of BRAF wild-type advanced melanoma: a cost-effectiveness analysis. Immunotherapy 10(14), 1241-1252 (2018).

-. This cost-effectiveness analysis demonstrated that, in BRAF wild-type melanoma, initiating treatment with anti-PD-1 + anti-CTLA-4 was cost effective compared with anti-PD-1 or anti-CTLA-4 initiating sequences.

27. Barzey V, Atkins MB, Garrison LP, Asukai Y, Kotapati S, Penrod JR. Ipilimumab in 2nd line treatment of patients with advanced melanoma: a cost-effectiveness analysis. J. Med. Econ. 16(2), 202-212 (2013).

28. Fleeman N, Bagust A, Beale S et al. Dabrafenib for treating unresectable, advanced or metastatic BRAF V600 mutation-positive melanoma: an evidence review group perspective. Pharmacoeconomics 33(9), 893-904 (2015).

29. Marriott E, Praet C, Aguiar-Ibáñez R et al. Cost-effectiveness of pembrolizumab for unresectable metastatic melanoma after progression with ipilimumab in England. Value Health 18(7), A453 (2015).

30. Beale S, Dickson R, Bagust A et al. Vemurafenib for the treatment of locally advanced or metastatic BRAF V600 mutation-positive malignant melanoma: a NICE single technology appraisal. Pharmacoeconomics 31(12), 1121-1129 (2013).

31. Wolchok JD, Chiarion-Sileni V, Gonzalez R et al. Overall survival with combined nivolumab and ipilimumab in advanced melanoma. N. Engl. J. Med. 377(14), 1345-1356 (2017).

32. Postow MA, Chesney J, Pavlick ACet al. Nivolumab and ipilimumab versus ipilimumab in untreated melanoma. N. Engl. J. Med. 372(21), 2006-2017 (2015).

33. Hodi FS, Chesney J, Pavlick AC et al. Combined nivolumab and ipilimumab versus ipilimumab alone in patients with advanced melanoma: 2-year overall survival outcomes in a multicentre, randomised, controlled, phase 2 trial. Lancet Oncol. 17(11), 1558-1568 (2016).

34. Atkins MB, McDermott D, Tarhini A et al. Matching-adjusted indirect comparison of nivolumab + ipilimumab and BRAF + MEK inhibitors for the treatment of $B R A F$-mutant treatment-naive advanced melanoma. Presented at: American Association for Cancer Research Annual Meeting. IL, USA, 14-18 April 2018 (Abstract 9538).

-. This analysis demonstrated that, after adjusting for baseline characteristics, the nivolumab + ipilimumab combination had a significant overall survival benefit over BRAF + MEK inhibitors for the treatment of patients with $B R A F$-mutant advanced melanoma.

35. Sullivan RJ, Atkins MB, Kirkwood JM et al. An update on the Society for Immunotherapy of Cancer consensus statement on tumor immunotherapy for the treatment of cutaneous melanoma: version 2.0. J. Immunother. Cancer 6(1), 44 (2018). 
36. NCCN guidelines for cutaneous melanoma V.1.2019 (2018). www.nccn.org/professionals/physician_gls/pdf/cutaneous_melanoma.pdf

37. Gibney GT, Atkins MB. Immunotherapy or molecularly targeted therapy: what is the best initial treatment for stage IV BRAF-mutant melanoma? Clin. Adv. Hematol. Oncol. 13(7), 451-458 (2015).

38. Firwana B, Sonbol MB, Atrash S et al. Efficacy of immunotherapy in advanced melanoma: a network meta-analysis. J. Clin. Oncol. 34(Suppl. 15), e21033 (2016).

39. Carretero-González A, Lora D, Ghanem I et al. Analysis of response rate with ANTI PD1/PD-L1 monoclonal antibodies in advanced solid tumors: a meta-analysis of randomized clinical trials. Oncotarget 9(9), 8706-8715 (2018).

40. Middleton MR, Grob JJ, Aaronson N et al. Randomized phase III study of temozolomide versus dacarbazine in the treatment of patients with advanced metastatic malignant melanoma. J. Clin. Oncol. 18(1), 158-166 (2000).

41. Hersh EM, O'Day SJ, Ribas A et al. A phase 2 clinical trial of nab-paclitaxel in previously treated and chemotherapy-naive patients with metastatic melanoma. Cancer 116(1), 155-163 (2010).

42. Larkin J, Minor D, D’Angelo S et al. Overall survival in patients with advanced melanoma who received nivolumab versus investigator's choice chemotherapy in CheckMate 037: a randomized, controlled, open-label phase III trial. J. Clin. Oncol. 36(4), 383-390 (2018).

43. OPDIVO $^{\circledR}$ (nivolumab), prescribing information. Bristol-Myers Squibb Company, NJ, USA (2015). https://packageinserts.bms.com/pi/pi_opdivo.pdf

44. KEYTRUDA ${ }^{\circledR}$ (pembrolizumab), prescribing information. Merck \& Co., Inc., NJ, USA (2017). www.merck.com/product/usa/pi_circulars/k/keytruda/keytruda_pi.pdf

45. YERVOY ${ }^{\circledR}$ (ipilimumab), prescribing information. Bristol-Myers Squibb Company, NJ, USA (2018). https://packageinserts.bms.com/pi/pi_yervoy.pdf

46. Hauschild A, Agarwala SS, Trefzer U et al. Results of a phase III, randomized, placebo controlled study of sorafenib in combination with carboplatin and paclitaxel as second line treatment in patients with unresectable stage III or stage IV melanoma. J. Clin. Oncol. 27(17), 2823-2830 (2009).

47. Truven Health Analytics. RED BOOK Online ${ }^{\circledR}$ (2016). http://truvenhealth.com/Products/Micromedex/Product-Suites/Clinical-Knowledge/RED-BOOK

48. Centers for Medicare \& Medicaid Services (CMS). Clinical Laboratory Fee Schedule (2018). www.cms.gov/Medicare/Medicare-Fee-for-Service-Payment/ClinicalLabFeeSched/index.html

49. Centers for Medicare \& Medicaid Services (CMS). Hospital Outpatient Prospective Payment System (2018). www.cms.gov/Medicare/Medicare-Fee-for-Service-Payment/HospitalOutpatientPPS/index.html

50. Bilir SP, Ma Q, Zhao Z, Wehler E, Munakata J, Barber B. Economic burden of toxicities associated with treating metastatic melanoma in the United States. Am. Health Drug Benefits 9(4), 203-213 (2016).

51. Agency for Healthcare Research and Quality (AHRQ). Healthcare Cost and Utilization Project (HCUP). National Inpatient Sample (NIS) (2018). www.hcup-us.ahrq.gov/db/nation/nis/nisdbdocumentation.jsp

52. Paly V, Colby C, Sabater J, Kotapati S. Predictors of utility over time among patients with treatment-naive advanced melanoma from the phase 3 CheckMate 067 trial. Presented at: International Society for Pharmacoeconomics and Outcomes Research 21st Annual International Meeting. DC, USA, 23-25 May 2016 (Abstract PCN130).

53. Brazier J, Rowen D. NICE DSU Technical Support Document 11: alternatives to EQ-5D for generating health state utility values (2011). http://nicedsu.org.uk/wp-content/uploads/2016/03/TSD11-Alternatives-to-EQ-5D_final.pdf

54. Beusterien KM, Szabo SM, Kotapati S et al. Societal preference values for advanced melanoma health states in the United Kingdom and Australia. Br. J. Cancer 101(3), 387-389 (2009).

55. Karnon J, Stahl J, Brennan A, Caro JJ, Mar J, Möller J. Modeling using discrete event simulation: a report of the ISPOR-SMDM Modeling Good Research Practices Task Force-4. Value Health 15(6), 821-827 (2012).

56. ClinicalTrials.gov. Sequential Combo Immuno and Target Therapy (SECOMBIT) Study (SECOMBIT) (2015). https://clinicaltrials.gov/ct2/show/NCT02631447

57. Kohn CG, Zeichner SB, Chen Q, Montero AJ, Goldstein DA, Flowers CR. Cost-effectiveness of immune checkpoint inhibition in $B R A F$ wild-type advanced melanoma. J. Clin. Oncol. 35(11), 1194-1202 (2017).

58. Neumann PJ, Cohen JT, Weinstein MC. Updating cost-effectiveness - the curious resilience of the $\$ 50,000$-per-QALY threshold. $N$. Engl. J. Med. 371(9), 796-797 (2014).

59. Johnson DB, Pectasides E, Feld E et al. Sequencing treatment in BRAFV600 mutant melanoma: anti-PD-1 before and after BRAF inhibition. J. Immunother. 40(1), 31-35 (2017). 